\title{
ON A THEOREM OF VON STERNECK
}

BY D. H. LEHMER*

1. Introduction. In 1894 von Sterneck $\dagger$ gave an interesting theorem which is essentially as follows.

THEOREM 1. Let $f$ and $g$ be any numerical functions and consider the function $h$ defined for all positive integers $n$ by the equa. tion

$$
h(n)=\sum_{n} f(i) g(j)
$$

the summation extending over all $(i, j)$ whose L.C.M. is $n$. Next write $F(n)=\sum f(\delta), G(n)=\sum g(\delta), H(n)=\sum h(\delta)$, where $\delta$ ranges over all the divisors of $n$. Then $F(n) G(n)=H(n)$.

It is the purpose of this note to supply the background for this theorem and to give a very simple proof of a theorem including among other special cases von Sterneck's theorem.

In a recent paper $\ddagger$ the author has considered the class of functions $\psi(x, y)$ which are subject to the following conditions or postulates.

Postulate A. If $x$ and $y$ are positive integers, so is $\psi(x, y)$.

Postulate I. For each integer $n>0$, the equation $\psi(x, y)=n$ has but a finite number of solutions $(x, y)$.

Postulate II. $\psi(x, y)=\psi(y, x)$.

Postulate III. $\psi(\psi(x, y), z)=\psi(x, \psi(y, z))$.

Postulate IV. $\psi(x, 1)=n$, implies $x=n$.

The solutions $x$ of $\psi(x, n)=n$ are called the associates of $n$. For the purposes of this paper we add the following postulate.

Postulate B. $\psi(x, y)$ is not an associate of $n$, when neither $x$ nor $y$ are associates of $n$.

* National Research Fellow.

$\dagger$ Monatshefte, vol. 5 (1894), p. 265. See also Dickson, History of the Theory of Numbers, vol. 1, p. 152. Von Sterneck's theorem is stated in terms of $k$ independent functions instead of 2 , as above. There is very little trouble in extending Theorem 1 and also Theorem 4 to this more general case. To avoid the consequent typographical complications, however, we leave this part to the reader.

$\ddagger$ Transactions of this Society, vol. 33 (1931). 
2. Examples of $\psi$-Functions. To each such $\psi$-function there corresponds a type of arithmetic of numerical functions. Only two $\psi$-functions are well known, and these lead to familiar branches of the theory of numbers. These functions are $x+y-1$ and $x y$. Much less familiar is the case $*(x, y)=[x, y]$, the L.C.M. of $x$ and $y$. Perhaps the simplest example of all is $\psi(x, y)$ $=\max (x, y)$. This case seems to have attracted no attention.

THEOREM 2. The most general polynomial satisfying the above postulates is

$$
A x y-(A-1)(x+y-1),
$$

where $A$ is a non-negative integer.

Proof. Any polynomial $P(x, y)$ must be symmetric by Postulate III. If $d$ is the degree of this polynomial in each variable, then comparing the degrees in $x$ of both sides of

$$
P(P(x, y), z)=P(x, P(y, z)),
$$

we have $d^{2}=d$. Now $d \neq 0$ because $\psi=$ constant fails to satisfy Postulate IV. Hence $d=1$, and $P(x, y)$ is bilinear. Writing

$$
P(x, y)=A x y+B(x+y)+C,
$$

and using Postulate IV we find that $B=1-A=-C$. Hence

$$
P(x, y)=A x y-(A-1)(x+y-1) .
$$

This polynomial satisfies the postulates if and only if $A$ is an integer $\geqq 0$.

3. Factorable $\psi$-Functions. Given a $\psi$-function, the fundamental theorem of arithmetic enables us to write down as many other $\psi$-functions as we please. The method is as follows. Every positive integer $n$ may be uniquely described by giving the exponents $n_{v}$ to which the $v$ th prime $p_{v}$ appears as a factor of $n=\prod p_{v}{ }^{n} v$ for $v=1,2,3, \cdots$. Let $\psi_{1}(x, y)$ be given. We define $\psi_{2}(x, y)$ by

$$
\psi_{2}(x, y)=\psi_{2}\left(\Pi p_{v} x_{v}, \Pi p_{v}^{y_{v}}\right)=\Pi p_{v}^{\psi_{1}\left(x_{v}+1, y_{v}+1\right)-1} .
$$

* Von Sterneck seems to have first used the function L.C.M. in this connection. The fundamentals of the L.C.M. calculus are developed in a recent paper, American Journal of Mathematics, vol. 53 (1931). See also a paper by E. T. Bell, this Bulletin, vol. 37 (1931), p. 85. 
Such a $\psi$-function is called factorable. As may be verified, $\psi_{2}$ satisfies the above postulates provided of course $\psi_{1}$ does also. Of the functions mentioned in $\$ 2$, only two are factorable, namely $x y$ and $[x, y]$, and these are derived from $x+y-1$ and $\max (x, y)$ respectively. These factorable functions in turn produce other factorable functions, namely, $\prod p_{v}{ }^{x_{v}+y_{v}+x_{v} y_{v} *}$ and $\prod p_{v}\left[x_{v}+1, y_{v}+1\right]-1$ and so on. The general polynomial $\psi$-function gives rise to $\Pi p_{v}{ }^{x_{v}+y_{v}+\boldsymbol{A} x_{v} y_{v}}$. The process may be reversed and carried back until a non-factorable function is reached.

Instead of representing $n$ as a product of powers of primes we may use its digits when written to the base 10 , for example. Thus $n=\sum_{r=0} n_{r} 10^{r}, 0 \leqq n_{r} \leqq 9$. From the function $\psi_{1}(x, y)$ $=\max (x, y)$ we derive

$$
\psi_{2}(x+1, y+1)=1+\sum_{r=0} \max \left(x_{r}, y_{r}\right) 10^{r} .
$$

4. The Main Theorem. We shall first prove the following theorem.

THEOREM 3. $\psi(x, y)$ is an associate of $n$ if and only if both $x$ and $y$ are associates of $n$.

Proof. Suppose first that both $x$ and $y$ are associates of $n$. Then by Postulate III,

$$
\psi(\psi(x, y), n)=\psi(x, \psi(y, n))=\psi(x, n)=n .
$$

Hence $\psi(x, y)$ is an associate of $n$. Next suppose that one variable, say $y$, is, and $x$ is not an associate of $n$. Then we can write

$$
\psi(\psi(x, y), n)=\psi(x, \psi(y, n))=\psi(x, n) \neq n .
$$

Hence $\psi(x, y)$ is, in this case, not an associate of $n$. Postulate B now completes the proof of the theorem. We now pass to the proof of the main theorem.

THEOREM 4. Let $f$ and $g$ be any numerical functions and consider the function $h$ defined for all positive integers $n$ by the equation

$$
h(n)=\sum_{n} f(i) g(j),
$$

* This function is not too complicated to give an interesting type of arithmetic, some details of which are given in the Transactions of this Society. The value of this function depends upon the multiplicative properties of exponents of the primes dividing $x$ and $y$. 
the summation extending over all $(i, j)$ for which $\psi(i, j)=n$. Next write $F(n)=\sum f(a), G(n)=\sum g(a), H(n)=\sum h(a)$, where a ranges over the associates of $n$. Then $F(n) G(n)=H(n)$.

PROOF. Let the function $\epsilon_{n}(m)$ have the value 1 or 0 according as $m$ is or is not an associate of $n$. Then if $x$ and $y$ are any positive integers,

$$
\epsilon_{n}(x) \epsilon_{n}(y)=\epsilon_{n}(\psi(x, y)) .
$$

In fact this follows at once from Theorem 3 . Hence we can write

$$
\begin{aligned}
F(n) G(n) & =\sum f(a) \sum g(a)=\sum_{x=1} f(x) \epsilon_{n}(x) \sum_{y=1} g(y) \epsilon_{n}(y) \\
& =\sum f(x) g(y) \epsilon_{n}(\psi(x, y)) \\
& =\sum_{m=1} \epsilon_{n}(m) \sum f(x) g(y), \quad(\psi(x, y)=m), \\
& =\sum_{m=1} \epsilon_{n}(m) h(m)=\sum h(a)=H(n),
\end{aligned}
$$

which is the theorem.

5. Examples of Theorem 4. In case $\psi(x, y)$ is a polynomial $P(x, y)$, the preceding theorem is trivial. In fact the equation $P(x, n)=n$ is linear in $x$ and hence has the single solution $x=1$ required by Postulate IV. That is, 1 is the only associate of $n$. Theorem 4 , in this case, simply states that $f(1) g(1)=h(1)$, which is (2) written for $n=1$. It may be shown that if $\psi_{1}$ is such that 1 is the only associate of $n,(n=1,2,3, \cdots)$, then the same is true of the factorable function $\psi_{2}$ defined by (1). Hence Theorem 4 is trivial for a $\psi$-function which is derived in this way from a polynomial.

Let $\psi(x, y)=\max (x, y)$. Then the associates of $n$ are $1,2,3, \cdots, n$. Hence if we write

$$
F(n)=\sum_{k=1}^{n} f(k), \quad G(n)=\sum_{k=1}^{n} g(k),
$$

Theorem 4 asserts that

$$
\sum_{k=1}^{n}\{f(k) G(k)+g(k) F(k)-f(k) g(k)\}=F(n) G(n) .
$$

In case $\psi(x, y)=[x, y]$, the associates of $n$ are its divisors. Theorem 4 then becomes von Sterneck's Theorem 1.

STANFORD UNIVERSITY 